\title{
Separation of Water and Ethanol by Pervaporation through Polymer Membranes Containing $N$-Substituted Imide Groups
}

\author{
Masakazu YoshIKawa, * Hideto YoKor, Kohei SANUI, \\ and Naoya OGATA
}

Department of Chemistry, Faculty of Science and Technology, Sophia University, 7-1 Kioi-cho, Chiyoda-ku, Tokyo 102, Japan

(Received April 17, 1984)

\begin{abstract}
Pervaporation of aqueous ethanol solution was carried out through three synthetic polymer membranes each containing an imide group, poly $(N$-phenylmaleimide-costyrene)s and a polymer having an $N$-substituted phthalimide group. These membranes selectively permeated water. The water was assumed to be permeated by a carrier mechanism utilizing a carbonyl moiety of the imide group as a carrier, while ethanol was permeated through membranes without specific interaction between ethanol and membranes.

KEY WORDS Pervaporation / Permselectivity / $N$-Substituted Imide Group / Carrier Mechanism / Michaelis-Menten Type Profile /
\end{abstract}

Separation technique by membranes are regarded as very promising means for energy saving conservation because of their high separation efficiency. The pervaporation technique is a potential method for specific and selective separation of neutral organic compounds such as organic mixtures.

In a previous paper, the authors reported the separation of water from aqueous alcohol solutions by pervaporation through poly(maleimide- $c o$-acrylonitrile), ${ }^{1-3}$ poly(maleimide-co-styrene), ${ }^{3}$ and poly(acrylic acid-coacrylonitrile $)^{4}$ membranes. In particular, poly(maleimide-co-acrylonitrile) and poly(acrylic acid-co-acrylonitrile) membranes gave high separation factors, exceeding 700 for water. The authors concluded that the high selectivity toward water through poly(maleimide-co-acrylonitrile) membrane was possible by the selective hydrogen-bonding interaction between water and maleimide units in the poly(maleimide-co-acrylonitrile) membrane. ${ }^{2}$ Not only the number of functional groups, which interact preferentially with water in the membrane, but also the environment (membrane polarity) surrounding the functional groups have been found to determine the separation characteristics of water-alcohol (ethanol) binary mixtures. ${ }^{3}$

In the present paper, attention is focussed on the $N$-substituted imide group as a carrier and poly $(N$-phenylmaleimide-co-styrene $) s$ and the polymer containing the $N$-substituted phthalimide group as a side chain. The permeation and separation of water-ethanol mixtures through these polymer membranes are discussed.

\section{EXPERIMENTAL}

\section{Materials}

$\mathrm{N}$-phenylmaleimide (1) was purified by recrystallization from cyclohexane solution and melted at $89.5-90.0^{\circ} \mathrm{C}$. Styrene (2) and 2,2'azobisisobutyronitrile (AIBN) were purified by the usual methods. Mixtures of 4- and 3- 
chloromethylstyrene (3) were distilled twice; bp, 80.7-80.9 C. Potassium phthalide (4), toluene, dimethyl sulfoxide (DMSO), tetrahydrofuran (THF), pyridine (Py), cyclohexanone, methanol, and ethanol were used as received without further purification. Water distilled twice was used in the present study.

\section{Syntheses of Polymers}

Compounds 1 and $\mathbf{2}$ in various molar ratios were placed in an ampule containing $0.18 \mathrm{~mol} \%$ AIBN and sealed after degassing. The bulk copolymerization was carried out at $52^{\circ} \mathrm{C}$ with shaking. The mixture was poured into methanol and filtered. The chemical composition of each polymer was determined by elemental analysis of carbon and nitrogen. Results of the copolymerizations are shown in Table I.

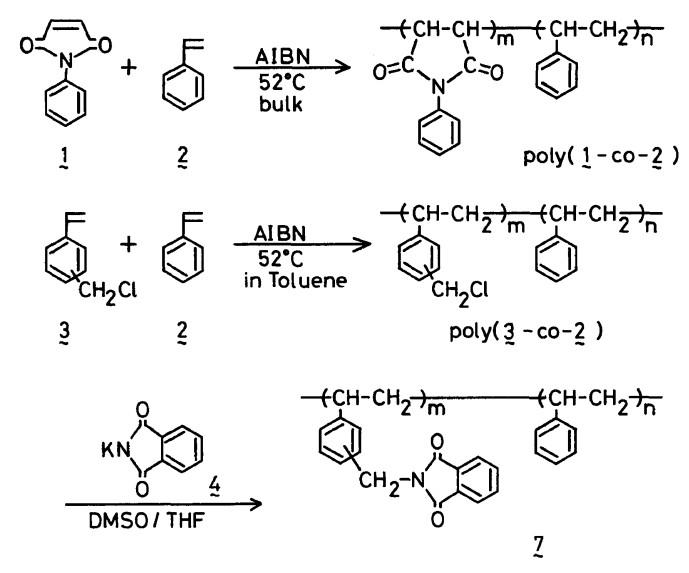

Polymer 7 was prepared as follows: a $2.15 \mathrm{~g}$ $\left(1.41 \times 10^{-2} \mathrm{~mol}\right)$ of $3,19.39 \mathrm{~g}(0.186 \mathrm{~mol})$ of 2 , and $137.4 \mathrm{mg}\left(1.01 \times 10^{-3} \mathrm{~mol}\right)$ of AIBN were placed in an ampule and the solution was sealed after degassing. The bulk copolymerization was carried out at $52^{\circ} \mathrm{C}$ for $40 \mathrm{~h}$ with shaking. The product was dissolved in toluene, and poured into methanol. Poly(3-co-2) was collected by filtration; yield, $17.30 \mathrm{~g}(80.33 \%)$. To $100 \mathrm{~cm}^{3}$ of THF solution containing $5.00 \mathrm{~g}$ of poly(3-co-2) was added $50 \mathrm{~cm}^{3}$ of DMSO solution containing $0.76 \mathrm{~g}\left(4.18 \times 10^{-3} \mathrm{~mol}\right)$ of 4. The mixture was refluxed at $90^{\circ} \mathrm{C}$ for $70 \mathrm{~h}$. The solution was poured into methanol and filtered. The precipitate was dissolved into THF and filtered to remove the precipitate. 7 was collected by pouring the THF solution into water, followed by filtration; yield, $4.83 \mathrm{~g}$ (90.28\% based on poly(3-co-2). The chemical composition of 7 was determined by a ${ }^{1} \mathrm{H}$ NMR spectrum recorded on a JEOL FX 200 (199.5 MHz).

\section{Preparation of Membranes}

The membranes were obtained by casting the polymer solutions. A $250 \mathrm{~g} \mathrm{dm}^{-3}$-Py solution of poly(1-co-2) and a $150 \mathrm{~g} \mathrm{dm}^{-3}$-cyclohexanone solution of 7 were poured onto glass plates using a glass rod and a ca. $0.15 \mathrm{~mm}$ spacer. The solvent was allowed to evaporate at $40^{\circ} \mathrm{C}$. The thickness of poly(1-co-2) membrane was $17-24 \mu \mathrm{m}$ and that of 7 membrane was $27-28 \mu \mathrm{m}$.

Table I. Results of copolymerization ${ }^{\mathrm{a}}$ of $\mathbf{1}$ and $\mathbf{2}$

\begin{tabular}{|c|c|c|c|c|c|c|c|c|c|}
\hline \multirow{4}{*}{ Polymer } & \multicolumn{2}{|c|}{ Monomer } & \multirow{4}{*}{$\frac{\mathrm{AIBN}}{\mathrm{mg}}$} & \multirow{4}{*}{$\frac{\text { Time }}{h}$} & \multirow{4}{*}{$\frac{\text { Yield }}{\mathrm{g}}$} & \multirow{4}{*}{$\frac{\text { Yield }}{\%}$} & \multirow{2}{*}{\multicolumn{2}{|c|}{ Mol fraction of $\mathbf{1}$ in }} & \multirow{4}{*}{$\frac{\eta_{\mathrm{sp}} / c^{\mathrm{b}}}{\mathrm{dm}^{3} \mathrm{~g}^{-1}}$} \\
\hline & \multirow{3}{*}{$\frac{1}{g}$} & \multirow{3}{*}{$\begin{array}{l}2 \\
\mathrm{~g}\end{array}$} & & & & & & & \\
\hline & & & & & & & Monomer & Polymer & \\
\hline & & & & & & & & & \\
\hline 5 & 0.606 & 51.74 & 122.6 & 12.3 & 9.42 & 18.00 & 0.007 & 0.045 & 0.0903 \\
\hline 6 & 0.433 & 51.84 & 122.6 & 4.0 & 3.08 & 5.89 & 0.005 & 0.152 & 0.1021 \\
\hline
\end{tabular}

a The bulk polymerization was carried out at $52^{\circ} \mathrm{C}$.

b Reduced viscosity was measured at $c=1.00 \mathrm{~g} \mathrm{dm}^{-3}$-Py at $30^{\circ} \mathrm{C}$. 


\section{Pervaporation}

Permeation of the water-ethanol mixture was carried out through membranes by an ordinary pervaporation technique. ${ }^{5}$ Each membrane area was about $12.6 \mathrm{~cm}^{2}$; the upstream volume was $200 \mathrm{~cm}^{3}$ and the downstream pressure was kept at about $400 \mathrm{~Pa}(3.0$ torr). Pervaporation experiments were carried out at constant temperature of $15^{\circ} \mathrm{C}$. The separation analysis was carried out with a HITACHI 023 Gas Chromatograph equipped with a 1-m-long column packed with Porapak Q.

The separation factor, $\alpha$, is defined as

$$
\alpha=\frac{Y_{\text {Water }} / Y_{\text {Ethanol }}}{X_{\text {Water }} / X_{\text {Ethanol }}}
$$

where the $Y_{i}$ 's are the weight fractions of permeates and the $X_{i}$ 's, those of the feeds, respectively.

\section{RESULTS AND DISCUSSION}

\section{Pervaporation through Poly(1-co-2) Mem- branes}

Figures 1(a) and 2(a) give the results of the pervaporation experiments, where the weight fractions of water in the permeates are plotted against those of the feeds. These experiments were carried out at $15^{\circ} \mathrm{C}$ through a poly(1-co2) membrane, whose mol fractions of 1 were 0.045 and 0.152 in the 5 and 6 membranes, respectively. These membranes showed the same tendency as preferentially allowing the permeation of water. In the region shown by broken lines, these two membranes were possibly swollen by ethanol. This swelling will be discussed in more detail below.

Figures 1(b) and 2(b) indicate the effects of feed composition on flux and the separation factor, $\alpha$, at $15^{\circ} \mathrm{C}$ of the 5 and 6 membranes, respectively. The fluxes of both membranes were around $4 \mathrm{~g} \mathrm{~m}^{-2} \mathrm{~h}^{-1}$, despite the change in feed composition. The $\alpha$ values through the 5 membrane increased with decreasing fraction of water in the feed to the

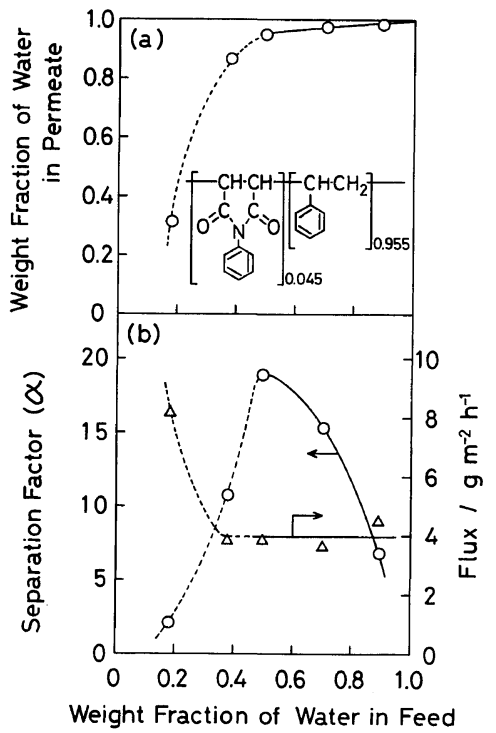

Figure 1. Effects of feed composition on the separation of water-ethanol, flux, and separation factor $(\alpha)$ in water-ethanol pervaporation through a poly $(1-c o-2)(5)$ membrane at $15^{\circ} \mathrm{C}$ ( 1 fraction, 0.045).

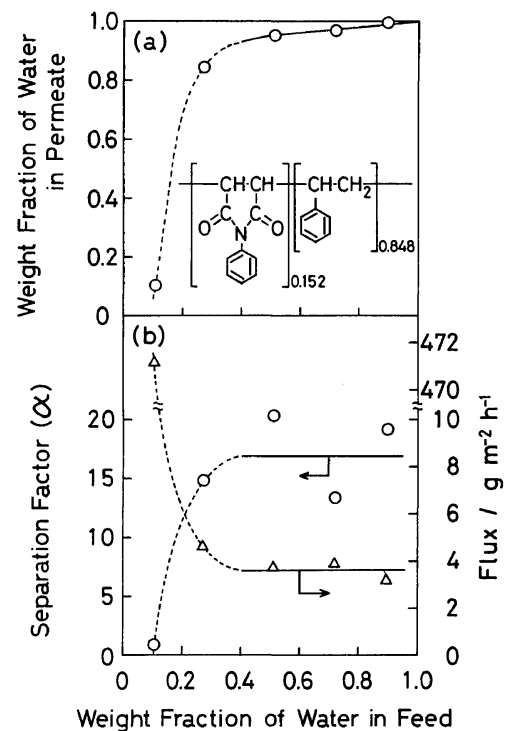

Figure 2. Effects of feed composition on the separation of water-ethanol, flux, and separation factor $(\alpha)$ in water-ethanol pervaporation through a poly(1-co-2) (6) membrane at $15^{\circ} \mathrm{C}$ (1 fraction, 0.152$)$.

point where the feed fraction was 0.5. A sudden fall in the $\alpha$ value was observed in the region shown by the broken lines, where a 
steep rise in the flux was also observed. From the profiles of $\alpha$ and flux shown in Figure 1(b), it was concluded that the $\mathbf{5}$ membrane swelled abruptly at this point. The swelling behavior was observed with a decrease in the weight fraction of water in the feed, that is, an increase in that of ethanol. As the weight fraction of ethanol in feed increased, ethanol entered the water layer where water was aggregated around imide groups by hydrogenbonding interaction. As a result, the membrane became swollen by ethanol. The $\alpha$ 's and fluxes through the 6 membrane showed profiles similar to those obtained by the 5 membrane despite the dispersion of the $\alpha$ values. Membrane 6 was also possibly swollen by ethanol in the region shown by the broken lines. To measure the swelling of the $\mathbf{5}$ and $\mathbf{6}$ membranes, membrane samples were immersed in various water-ethanol mixtures and the changes studied. The treated membranes showed the same weight, area, and thickness as the original membranes within experimental error. Thus, membrane swelling could not be considered as a numerical value even in a region shown by broken lines. The same results were obtained for the 7 membrane.

\section{Pervaporation through 7 Membrane}

Figure 3 indicates the results of the pervaporation experiments, where the weight fractions of water in the permeates, fluxes, and separation factor $(\alpha)$ are plotted against weight fractions of water in the feed. This membrane also preferentially permeated water as was observed in the poly(1-co-2) membrane system. The fluxes were held at a constant value, about $3 \mathrm{~g} \mathrm{~m}^{-2} \mathrm{~h}^{-1}$, while the $\alpha$ values increased with decreasing water fraction in the feed. At the point where the feed composition was around 0.1 , however, a steep rise in the flux was observed and the $\alpha$ value dropped considerably in response to the increase in flux number. These phenomena may also be attributed to membrane swelling.

Comparing the $\alpha$ values of the poly(1-co-2)

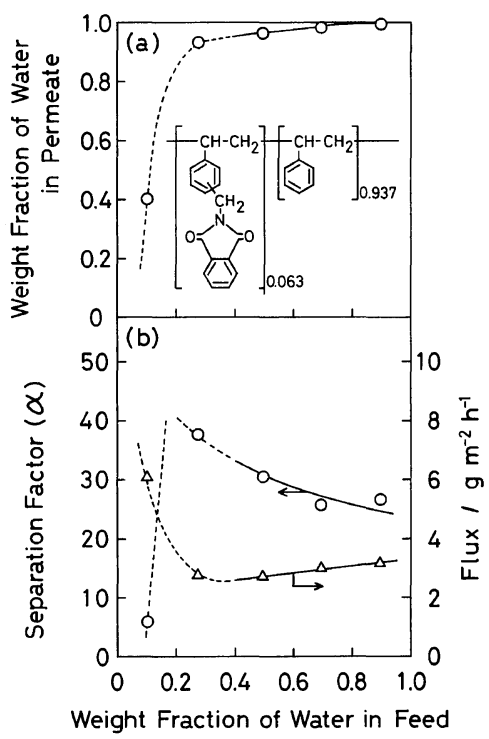

Figure 3. Effects of feed composition on the separation of water-ethanol, flux, and separation factor $(\alpha)$ in water-ethanol pervaporation through the 7 membrane at $15^{\circ} \mathrm{C}(\mathrm{N}$-substituted phthalimide fraction, 0.063$)$.

and 7 membranes, those of the 7 membrane were higher. This may be due to the following reasons: the hydrogen-bonding between water and the carbonyl moieties which are considered to be potential binding sites for water, ${ }^{2}$ in the 7 membrane is thought to be strengthened by the hydrophobicity of two benzene rings around the carbonyl moieties. The amount of water in the 7 membrane is thought to be more than that in 5 or $\mathbf{6}$ membrane. Thus, the 7 membrane preferentially permeates more water than the poly(1-co-2), 5 and 6 membranes.

\section{Permeation Mechanism}

Our previous experiment ${ }^{2}$ based on a spectroscopic approach was carried out by the selective hydrogen-bonding interaction between water and a maleimide unit in a poly(maleimide-co-acrylonitrile) membrane. In the present paper, we separated observed fluxes into those of ethanol and water and each separated flux was plotted against its concentration in the feed. The fluxes for the 5, $\mathbf{6}$, and 7 membranes are shown in Figures 4, 5, 

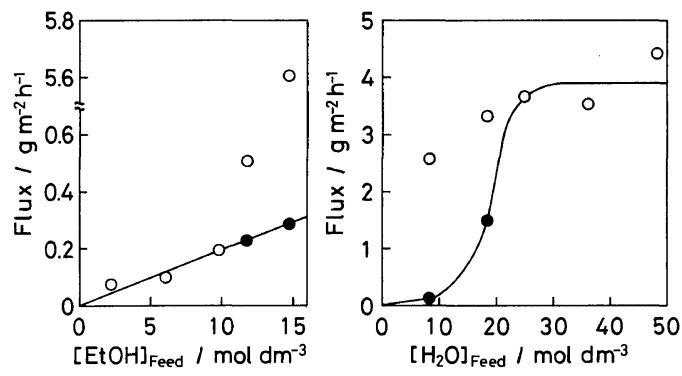

Figure 4. Relationship between ethanol (left) and water (right) fluxes of pervaporation through the $\mathbf{5}$ membrane, and their concentration in the feed $(O$, observed flux; $\mathbf{O}$, corrected flux).
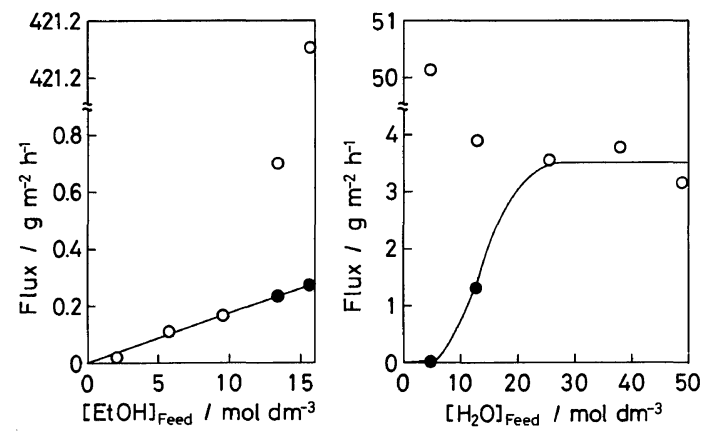

Figure 5. Relationship between ethanol (left) and water (right) fluxes of pervaporation through the 6 membrane, and their concentration in the feed $(O$, observed flux; $\mathbf{O}$, corrected flux).
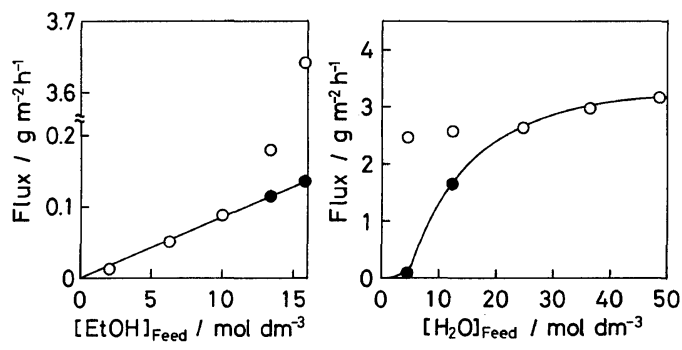

Figure 6. Relationship between ethanol (left) and water (right) fluxes of pervaporation through the 7 membrane, and their concentration in the feed $(O$, observed flux; - corrected flux).

and 6 , respectively.

These three membranes had similar profiles. First, the relationship between the ethanol flux and ethanol concentration in the feed was investigated and the results are shown in the left part of Figures 4, 5, and 6. The low concentration region, below $10 \mathrm{~mol} \mathrm{dm}^{-3}$ gave a straight line passing through the origin. This implies that ethanol was permeated through these three membranes without any specific interaction occurring between them. A straight line was not observed in the high concentration region of over $10 \mathrm{~mol} \mathrm{dm}^{-3}$. This deviation was considered due to the swelling of the membrane.

The relationship between water flux and concentration in the feed can be seen in the right of Figures $4-6$. The observed water fluxes are shown by open plots in these figures. It was difficult to find a clear relationship from these values. The effect of membrane swelling must be eliminated in order to evaluate membrane permeation properties. Ethanol fluxes in the left of the figures allowed correction for swelling effect. Assuming the fluxes obtained by the non-swelling membranes to obey the solid lines in the left of the figures, ethanol fluxes deviating from the straight lines were made drop coincide with the line. The closed plots are the corrected values in the relation between ethanol fluxes and concentrations. The corrected water fluxes were calculated by the following equations.

\section{Corrected Water Flux \\ $=$ Observed Water Flux $\times($ Corrected Ethanol Flux/ \\ Observed Ethanol Flux)}

The corrected water fluxes were also plotted by the closed plots in the relationship between flux and water concentration in feed. The corrected fluxes showed the water fluxes to reach a limited rate with increasing water concentration. The fact that fluxes showed Michaelis-Menten type profiles confirms that water is transported by a carrier mechanism. A carbonyl moiety may be a functional group for selective interaction with water, i.e., a fixed carrier for water. 


\section{CONCLUSIONS}

Water was preferentially permeated from an aqueous ethanol solution through the 5, 6, and 7 membranes via interaction with carbonyl moieties in the imide groups. Thus, water may be permeated by a carrier mechanism but ethanol through membranes without specific interaction with the membranes. This difference is responsible for the high selectivity for water.

Acknowledgement. This work was sup- ported by a Grant-in-Aid for Scientific Research from the Ministry of Education, Science, and Culture of Japan.

\section{REFERENCES}

1. M. Yoshikawa, H. Yokoi, K. Sanui, and N. Ogata, J. Polym. Sci., Polym. Lett. Ed., 22, 125 (1984).

2. M. Yoshikawa, H. Yokoi, K. Sanui, and N. Ogata, $J$. Polym. Sci., Polym. Chem. Ed., 22, 2159 (1984).

3. M. Yoshikawa, H. Yokoi, K. Sanui, N. Ogata, and T. Shimidzu, Polym. J., 16, 653 (1984).

4. M. Yoshikawa, T. Yukoshi, K. Sanui, and N. Ogata, J. Polym. Sci., Polym. Lett. Ed., 22, 473 (1984).

5. S. Yamada, Maku, 6, 168 (1981). 